\title{
An older patient with diabetes and severe foot pain
}

\author{
Gary Ross Farris MD, Starr Steinhilber MD MPH
}

cite as: CMAJ 2017 January 16;189:E73-5. doi: 10.1503/cmaj.160366

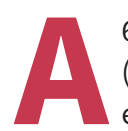
69-year-old man with a history of chronic kidney disease (stage 2), diabetic neuropathy and chronic venous stasis edema presented to his primary care clinic with a fourmonth history of progressive pain and swelling of the left foot. The pain had become more intense after a minor trauma six weeks earlier. His entire left foot was erythematous with exquisite tenderness over the dorsal and plantar midfoot and forefoot with no skin breakdown, tophi or effusions. Foot radiography showed a periosteal reaction and thinning of the lateral aspect of the second metatarsal bone. His serum uric acid level was 583 (normal 149-446) $\mu \mathrm{mol} / \mathrm{L}$.

Gout was diagnosed, and the patient was prescribed an oral corticosteroid with a tapered dosage over five days. Magnetic resonance imaging (MRI), ordered owing to nonresolution of symptoms, showed erosive changes, which raised concerns about osteomyelitis (Figure 1). Erythrocyte sedimentation rate was 82 (normal $0-15$ ) $\mathrm{mm} / \mathrm{h}$ and C-reactive protein level was 70.5 (normal < 8.0) $\mathrm{mg} / \mathrm{L}$.

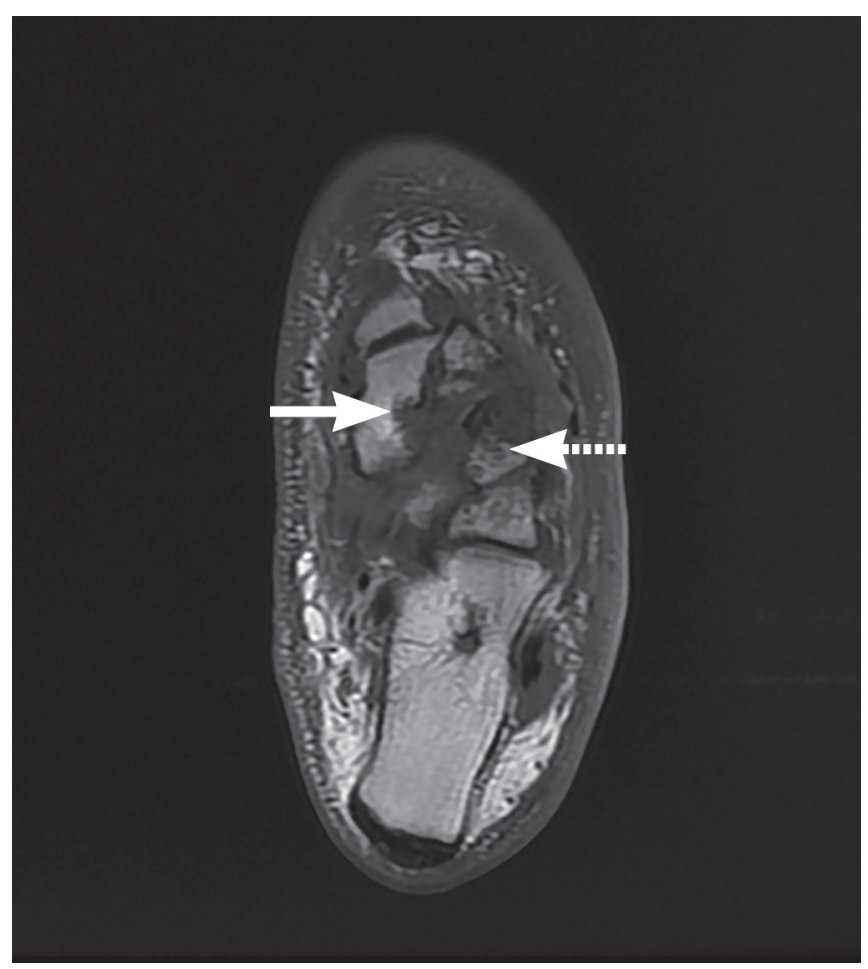

Figure 1: Magnetic resonance image showing diffuse soft-tissue edema throughout the foot with loss of cortex (dashed arrow) and erosive changes (solid arrow) of cuneiform bones. This, along with a periosteal reaction along the second metatarsal bone, raised concerns about osteomyelitis.
The patient was admitted to hospital with a presumptive diagnosis of osteomyelitis. Samples were drawn for blood culture, and he was given empiric antibiotics (intravenous vancomycin and cefepime), because of concern about polymicrobial infection. Arthrocentesis was not possible owing to the absence of an effusion.

The patient was discharged home with a peripherally inserted central catheter and instructions to continue the intravenous antibiotics for six weeks. Four days after discharge, he returned to the hospital with worsening symptoms, including newly inflamed proximal and distal interphalangeal joints on both hands, fever of $102.9^{\circ} \mathrm{F}$ $\left(39.4^{\circ} \mathrm{C}\right)$, tachycardia to 116 beats/min and an increase in serum creatinine level from 106 at baseline to 230 (normal 62-133) $\mu \mathrm{mol} / \mathrm{L}$. Repeat blood cultures were done. Vancomycin was discontinued owing to a possible contribution to the new acute kidney injury, and daptomycin was started.

Following this change, the patient's creatinine level returned to his baseline level; however, his fever and joint pain persisted. Both initial and repeat blood cultures remained negative. The following day coincided with a change in the patient's medical care team, and we re-evaluated the case in detail, given a lack of improvement despite aggressive therapy.

\section{What is the next best step?}

a. Consult the surgical service for a bone biopsy

b. Repeat blood cultures and continue antibiotic therapy

c. Reimage the affected foot

d. Stop all antibiotics owing to the lack of improvement and negative cultures

Because of the lack of improvement in the original joint symptoms and continued fever despite broad-spectrum antibiotic therapy, we decided to revisit the working diagnosis of osteomyelitis. Bone biopsy (a) could have confirmed a diagnosis of osteomyelitis or suggested an alternative diagnosis, but there were less invasive options. The utility of additional blood cultures (b) was limited given that multiple cultures were already negative. Stopping all antibiotics (d) would have been a reasonable option given the patient's stable vital signs, but this alone would not clarify a diagnosis. Our team reviewed the existing images with a radiologist, who thought that, although the most likely diagnosis was osteomyelitis, a crystal arthropathy could not be ruled out. Additional imaging of the foot (c) was therefore the most reasonable next step. 


\section{What follow-up imaging would be most helpful?}
a. Repeat MRI
b. Computed tomography (CT) with and without contrast
c. Dual-energy CT (DECT)
d. Ultrasonography

Magnetic resonance imaging (a) is the imaging test of choice for diagnosing bacterial osteomyelitis because of its sensitivity and high negative predictive value. Even so, it can be difficult to differentiate osteomyelitis from other diagnoses based on MRI alone. A recent meta-analysis by Khodaee and colleagues ${ }^{1}$ suggested that the sensitivity of MRI for bacterial osteomyelitis was $90 \%$ and its specificity was $79 \%$. The decreased specificity is because neuropathic arthropathy, contusion, fracture, malignancy and other arthritides can all present with bone marrow edema that is classically associated with bacterial osteomyelitis. ${ }^{2}$ For this reason, repeat MRI was not the best choice. Conventional CT (b) can be useful when MRI cannot be obtained because of a patient's implanted hardware interacting with magnetic and radio frequency fields. It has utility in diagnosing osteomyelitis, but it does not usually allow the distinction between various soft-tissue deposits to accurately diagnose crystal arthropathy. ${ }^{3}$ Ultrasonography (d) has some utility in gout, especially in identifying fluid collections for arthrocentesis, but Carter and colleagues ${ }^{4}$ suggest that ultrasonography is less sensitive than MRI in diagnosing gout. Dual-energy CT is a form of imaging that allows uric acid to be identified and differentiated from calcium in bone and tissue. ${ }^{5}$ Multiple studies of DECT have shown that it improved diagnostic accuracy of gout. ${ }^{6}$ Because of this, DECT (c) is the most appropriate next imaging choice.

Unfortunately, DECT was unavailable owing to technical problems. Conventional CT of the left foot showed diffuse tophaceous gout (Figures 2 and 3). We discontinued all antibiotics.

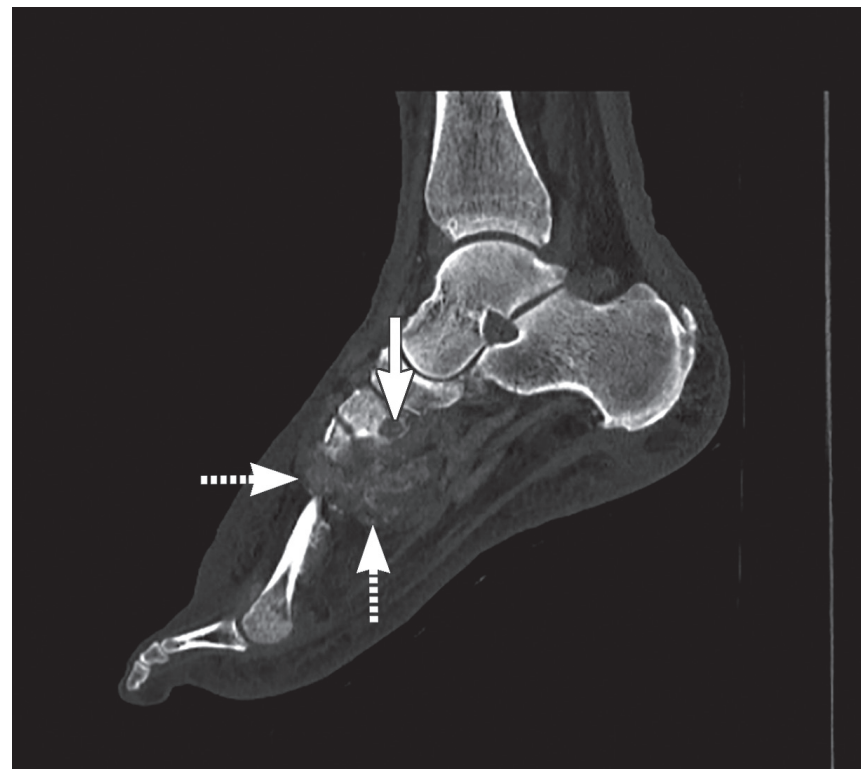

Figure 2: Computed tomography image of the left foot showing profound diffuse amorphous calcifications (dashed arrows). This, coupled with the bony erosion (solid arrow), is consistent with diffuse tophaceous gout.

\section{What is the best treatment for this patient?}

a. Indomethacin $50 \mathrm{mg}$ orally every 8 hours

b. Prednisone $40 \mathrm{mg}$ daily

c. Allopurinol $100 \mathrm{mg}$ daily

d. Prednisone, colchicine and allopurinol daily

Answer (d) is correct. Guidelines from the American College of Rheumatology state that combination therapy of either nonsteroidal anti-inflammatory drugs (NSAIDs) and colchicine, oral corticosteroids and colchicine, or intra-articular steroids with an oral agent are recommended for severe flares of gout. ${ }^{7}$ The guidelines defined severe gout flares by a pain rating of 7 or greater on a 0-10 visual analogue scale in patients with acute polyarthritis. ${ }^{7}$ It was previously thought that allopurinol and other urate-lowering therapies should not be started during an acute gout flare for fear of causing transient hyperuricemia and worsening the flare. More recent literature suggests that this risk is often overstated. ${ }^{7,8}$ As long as appropriate anti-inflammatory agents are in place, uratelowering therapy may be initiated during or immediately after an acute gout attack. ${ }^{8}$ Nonsteroidal anti-inflammatory drugs (a) should not be used in this patient because of his chronic kidney disease and recent acute kidney injury.

We chose pharmacologic urate-lowering therapy with colchicine $1.2 \mathrm{mg}$ once, then $0.6 \mathrm{mg}$ daily, and allopurinol $100 \mathrm{mg}$ daily, based on the indications in Box 1. In addition, we prescribed intravenous methylprednisolone for three days, then prednisone $40 \mathrm{mg}$ daily. The patient's gout improved rapidly.

\section{Discussion}

Clinical decision-making among physicians is a complex process involving both intuitive and analytical skills. In addition to the review of imaging studies and treatment above, this case high-

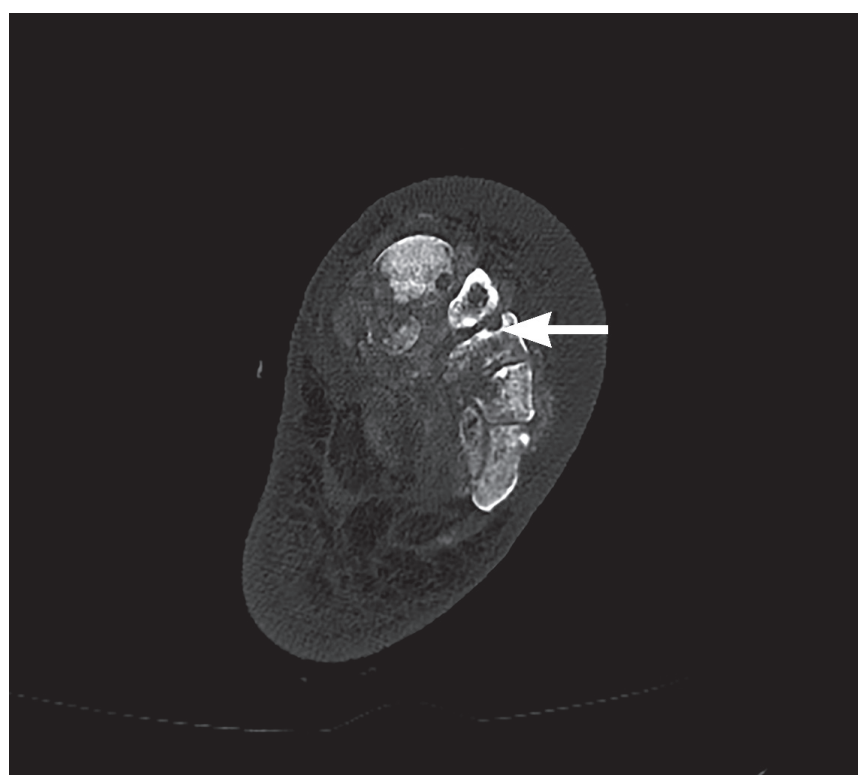

Figure 3: Computed tomography image of the midfoot also shows profound tophaceous gout and bony erosion. The arrow highlights the bony erosion. 
Box 1: Indications for pharmacologic urate-lowering therapy ${ }^{7}$

Established diagnosis of gouty arthritis and

- Tophus or tophi established by clinical examination or imaging

- Two or more attacks per year of acute gouty arthritis

- Chronic kidney disease stage 2 or worse

- History of urolithiasis

lights cognitive errors for which physicians are at risk when making clinical decisions. The clinical-reasoning concept of "illness scripts" and cognitive errors of anchoring and diagnosis momentum $^{9,10}$ are illustrated in this case.

An illness script is a summary of a diagnosis and includes predisposing factors, pathophysiology and clinical findings. ${ }^{9} \mathrm{~A}$ warm erythematous joint in a patient with diabetes and recent trauma can fit into a clinician's illness script of osteomyelitis. The fear of complications from untreated osteomyelitis understandably caused the primary team to err on the side of antibiotic therapy; however, when the patient returned without clinical improvement of his foot despite intravenous antibiotic therapy, the team adjusted the antibiotics rather than changing the treatment approach. This is an example of anchoring and diagnosis momentum. Anchoring bias is locking on to a diagnosis too early and failing to adjust the diagnosis as contradicting (or nonsupporting) information presents itself. ${ }^{10}$ Closely related is diagnosis momentum, which occurs when a previous diagnosis is accepted without sufficient review of material or skepticism. ${ }^{10}$ The treatment approach, consistent with a diagnosis of osteomyelitis, was not adjusted despite contradictory clinical evidence in the form of continued pain, erythema and swelling after broad antibiotic treatment. The team initially relied heavily on the MRI findings, although the positive predictive value is not high, as previously discussed. Subsequently, there was anchoring on the diagnosis of osteomyelitis, and the diagnosis was perpetuated even though the patient's condition was not improving as expected (diagnosis momentum).

All physicians are at risk for cognitive errors. It is important to consider all of the information available when making a clinical decision and to consciously re-evaluate a diagnosis when the clini- cal course does not fit with what is expected. Additionally, sharing a complex case with colleagues, such as the radiologist or new team in our case, can help uncover cognitive errors and biases early and expedite correct diagnosis and treatment.

\section{References}

1. Khodaee M, Lombardo D, Montgomery L, et al. What's the best test for underlying osteomyelitis in patients with diabetic foot ulcers? J Fam Pract 2015;64: 309-10, 321.

2. Tomas MB, Patel M, Marwin SE, et al. The diabetic foot. Br J Radiol 2000;73: 443-50.

3. Nicolaou S, Yong-Hing J, Galea-Soler S, et al. Dual-energy CT as a potential new diagnostic tool in the management of gout in the acute setting. AJR Am J Roentgenol 2010;194:1072-8.

4. Carter JD, Kedar R, Anderson S, et al. An analysis of MRI and ultrasound imaging in patients with gout who have normal plain radiographs. Rheumatology 2009;48:1442-6.

5. McQueen FM, Reeves Q, Dalbeth N. Advanced imaging in the diagnosis and management of gout. Postgrad Med J 2013;89:87-93.

6. Finkenstaedt T, Manoliou A, Toniolo M, et al. Gouty arthritis: the diagnostic and therapeutic impact of dual-energy CT. Eur Radiol 2016;26:3989-99.

7. Khanna D, Fitzgerald JD, Khanna PP, et al. 2012 American College of Rheumatology guidelines for management of gout. Part 1: systematic nonpharmacologic and pharmacologic therapeutic approaches to hyperuricemia. Arthritis Care Res (Hoboken) 2012;64:1431-46.

8. Shmerling RH. Management of gout: a 57-year-old man with a history of podagra, hyperuricemia, and mild renal insufficiency. JAMA 2012;308:2133-41.

9. Charlin B, Boshuizen HP, Custers EJ, et al. Scripts and clinical reasoning. Med Educ 2007;41:1178-84.

10. Croskerry $\mathrm{P}$. The importance of cognitive errors in diagnosis and strategies to minimize them. Acad Med 2003;78:775-80.

\section{Competing interests: None declared.}

This article has been peer reviewed.

The authors have obtained patient consent.

Affiliations: UAB Tinsley Harrison Internal Medicine Residency Program (Farris, Steinhilber); Birmingham Veterans Affairs Medical Center (Steinhilber), Birmingham, Ala.

Contributors: Both authors contributed to the conception and design of the work, drafted and revised the article, gave final approval of the version to be published and agreed to act as guarantors of the work.

Correspondence to: Gary Farris, grfarris1@uabmc.edu 